\title{
MULTIPLICITY RESULTS FOR FRACTIONAL LAPLACE PROBLEMS WITH CRITICAL GROWTH
}

\author{
ALESSIO FISCELLA, GIOVANNI MOLICA BISCI, AND RAFFAELLA SERVADEI
}

\begin{abstract}
This paper deals with multiplicity and bifurcation results for nonlinear problems driven by the fractional Laplace operator $(-\Delta)^{s}$ and involving a critical Sobolev term. In particular, we consider

$$
\begin{cases}(-\Delta)^{s} u=\gamma|u|^{2^{*}-2} u+f(x, u) & \text { in } \Omega \\ u=0 & \text { in } \mathbb{R}^{n} \backslash \Omega,\end{cases}
$$

where $\Omega \subset \mathbb{R}^{n}$ is an open bounded set with continuous boundary, $n>2 s$ with $s \in(0,1)$, $\gamma$ is a positive real parameter, $2^{*}=2 n /(n-2 s)$ is the fractional critical Sobolev exponent and $f$ is a Carathéodory function satisfying different subcritical conditions.
\end{abstract}

\section{INTRODUCTION}

Recently, the interest towards nonlocal fractional Laplacian equations involving a critical term has grown more and more. Concerning the existence result for this kind of problems, a positive answer has been given in the recent papers [3, 10, 13, 16, 22, 20]: in all these works well known existence results for classical Laplace operators were extended to the nonlocal fractional setting. A natural question is to ask when it is possible to get more than a non-trivial solution, giving a multiplicity result. In literature few attempts have been made to answer this question. In particular we refer to very recent papers [8, 15] which give a bifurcation result.

Motivated by the above papers, here we deal with the following problem

$$
\begin{cases}(-\Delta)^{s} u=\gamma|u|^{2^{*}-2} u+f(x, u) & \text { in } \Omega \\ u=0 & \text { in } \mathbb{R}^{n} \backslash \Omega,\end{cases}
$$

where $s \in(0,1)$ is fixed, $n>2 s, \Omega \subset \mathbb{R}^{n}$ is an open and bounded set with continuous boundary, $2^{*}=2 n /(n-2 s)$ and $(-\Delta)^{s}$ is the fractional Laplace operator, that may be defined (up to a normalizing constant) by the Riesz potential as follows

$$
(-\Delta)^{s} u(x)=\int_{\mathbb{R}^{n}} \frac{2 u(x)-u(x+y)-u(x-y)}{|y|^{n+2 s}} d y, \quad x \in \mathbb{R}^{n},
$$

as defined in [7] (see this paper and the references therein for further details on fractional Laplacian).

Concerning the nonlinearity in (1.1), in the present work we assume that $f: \Omega \times \mathbb{R} \rightarrow \mathbb{R}$ is a Carathéodory function satisfying the following condition

$$
\sup \{|f(x, t)|: \quad x \in \Omega,|t| \leqslant M\}<+\infty \text { for any } M>0 .
$$

Key words and phrases. Fractional Laplacian, critical nonlinearities, best fractional critical Sobolev constant, variational techniques, integrodifferential operators.

2010 AMS Subject Classification: Primary: 49J35, 35A15, 35S15; Secondary: 47G20, 45 G05.

The first author was supported by Coordenação de Aperfeiçonamento de pessoal de nível superior (CAPES) through the fellowship 33003017003P5-PNPD20131750-UNICAMP/MATEMÁTICA. The second and the third author were supported by the INdAM-GNAMPA Project 2016 Problemi variazionali su varietà riemanniane e gruppi di Carnot, by the DiSBeF Research Project 2015 Fenomeni non-locali: modelli e applicazioni and by the DiSPeA Research Project 2016 Implementazione e testing di modelli di fonti energetiche ambientali per reti di sensori senza fili autoalimentate. The third author was supported by the ERC grant $\epsilon$ (Elliptic Pde's and Symmetry of Interfaces and Layers for Odd Nonlinearities). 
The main aim of the present paper is to establish bifurcation results for (1.1). For this, we need that $f(x, t)$ is odd in $t$, i.e.

$$
f(x, t)=-f(x,-t) \quad \text { for any } t \in \mathbb{R} \text { and a.e. } x \in \Omega,
$$

in order to apply the symmetric version of the Mountain Pass Theorem due to Ambrosetti and Rabinowitz (see [2]). However, with respect to the classical case presented in [2], we use a weaker condition than the usual one of Ambrosetti-Rabinowitz, in order to overcome the lack of compactness at critical level $L^{2^{*}}(\Omega)$. Thus, we assume that $f$ and its primitive $F$, defined as

$$
F(x, t)=\int_{0}^{t} f(x, \tau) d \tau
$$

satisfy

$$
\begin{gathered}
\lim _{|t| \rightarrow+\infty} \frac{f(x, t)}{|t|^{2^{*}-1}}=0 \text { uniformly a.e. in } \Omega ; \\
\text { there exist } \sigma \in[0,2) \text { and } a_{1}, a_{2}>0 \text { such that } \\
\frac{1}{2} f(x, t) t-F(x, t) \geqslant-a_{1}-a_{2}|t|^{\sigma} \text { for any } t \in \mathbb{R} \text { and a.e. } x \in \Omega ; \\
\text { there exist } \theta \in\left(2,2^{*}\right) \text { and } b_{1}, b_{2}>0 \text { such that } \\
F(x, t) \leqslant b_{1}|t|^{\theta}+b_{2} \text { for any } t \in \mathbb{R} \text { and a.e. } x \in \Omega ; \\
\text { there exist } c_{1}>0, h_{1} \in L^{1}(\Omega) \text { and } \Omega_{0} \subset \Omega \text { with }\left|\Omega_{0}\right|>0 \text { such that } \\
F(x, t) \geqslant-h_{1}(x)|t|^{2}-c_{1} \text { for any } t \in \mathbb{R} \text { and a.e. } x \in \Omega \text { and } \\
\liminf _{|t| \rightarrow+\infty} \frac{F(x, t)}{|t|^{2}}=+\infty \text { uniformly a.e. in } \Omega_{0} .
\end{gathered}
$$

We are now ready to state our first result.

Theorem 1.1. Let $s \in(0,1), n>2 s, \Omega$ be an open bounded subset of $\mathbb{R}^{n}$ with continuous boundary, and let $f$ be a function satisfying assumptions (1.3), (1.4), (1.6), (1.7)-(1.9).

Then, for any $k \in \mathbb{N}$ there exists $\gamma_{k} \in(0,+\infty]$ such that (1.1) admits at least $k$ pairs of non-trivial solutions for any $\gamma \in\left(0, \gamma_{k}\right)$.

In the next result we establish a multiplicity result of solutions for (1.1) without assuming that primitive $F$ still satisfies a general subcritical growth like in (1.8). However, we need a stronger condition than (1.9). That is, given $j, k \in \mathbb{N}$ with $j \leqslant k$, we consider these different versions of (1.8) and (1.9)

$$
\begin{gathered}
\text { there exists a measurable function } a: \Omega \rightarrow \mathbb{R} \text { such that } \\
\limsup _{t \rightarrow 0} 2 \frac{F(x, t)}{|t|^{2}}=a(x) \text { uniformly a.e. in } \Omega, \\
a(x) \leqslant \lambda_{j} \text { a.e. in } \Omega \text { and } a(x)<\lambda_{j} \text { on a set of positive measure contained in } \Omega ; \\
\text { there exists } B>0 \text { such that } \\
F(x, t) \geqslant \lambda_{k} \frac{|t|^{2}}{2}-B \text { for any } t \in \mathbb{R} \text { and a.e. } x \in \Omega,
\end{gathered}
$$

where $\lambda_{j} \leqslant \lambda_{k}$ are eigenvalues of $(-\Delta)^{s}$, as recalled in Section 4 ,

With the above conditions we still can apply the Mountain Pass Theorem given in [2], getting the following result:

Theorem 1.2. Let $s \in(0,1), n>2 s, \Omega$ be an open bounded subset of $\mathbb{R}^{n}$ with continuous boundary. Let $j, k \in \mathbb{N}$, with $j \leqslant k$, and let $f$ be a function satisfying assumptions (1.3), (1.4), (1.6), (1.7), (1.10) and (1.11).

Then, there exists $\gamma_{k, j} \in(0,+\infty]$ such that (1.1) admits at least $k-j+1$ pairs of nontrivial solutions for any $\gamma \in\left(0, \gamma_{k, j}\right)$. 
A natural question is to investigate what happens when $f$ has not any symmetry. In this case it is still possible to get a multiplicity result, by studying two truncated problems related to (1.1). These auxiliary problems are still variational and by using the Mountain Pass Theorem we get at least two solutions of different sign for them, as stated in the following result:

Theorem 1.3. Let $s \in(0,1), n>2 s, \Omega$ be an open bounded subset of $\mathbb{R}^{n}$ with continuous boundary. Let $f$ satisfy $f(x, 0)=0$, (1.3), (1.6), (1.7), (1.10) and (1.11) with $j=k=1$.

Then, there exists $\gamma_{1}>0$ such that (1.1) admits a non-trivial non-negative and a nontrivial non-positive solution for any $\gamma \in\left(0, \gamma_{1}\right)$.

The main tools used in order to prove Theorem 1.1-Theorem 1.3 are variational and topological methods and a suitable decomposition of the functional space $X_{0}^{s}(\Omega)$ where we look for solutions of problem (1.1), through the eigenvalues of the fractional Laplace operator.

An interesting open problem is to prove the main results of the present paper in a more general framework, like the one given in the following problem:

$$
\begin{cases}-\mathcal{L}_{K}^{p} u=\gamma|u|^{p^{*}-2} u+f(x, u) & \text { in } \Omega \\ u=0 & \text { in } \mathbb{R}^{n} \backslash \Omega,\end{cases}
$$

where $\Omega \subset \mathbb{R}^{n}$ is an open and bounded set with continuous boundary, $n>p s \geqslant 2 s$, $p^{*}=p n /(n-p s)$ and $\mathcal{L}_{K}^{p}$ is a nonlocal operator defined as follows:

$$
\mathcal{L}_{K}^{p} u(x)=2 \lim _{\varepsilon \searrow 0} \int_{\mathbb{R}^{n} \backslash B_{\varepsilon}(x)}|u(x)-u(y)|^{p-2}(u(x)-u(y)) K(x-y) d y, \quad x \in \mathbb{R}^{n} .
$$

Here, the kernel $K: \mathbb{R}^{n} \backslash\{0\} \rightarrow(0,+\infty)$ is a measurable function for which

$$
m K \in L^{1}\left(\mathbb{R}^{n}\right) \text {, with } m(x)=\min \left\{|x|^{p}, 1\right\} ;
$$

$$
\text { there exists } \theta>0 \text { such that } K(x) \geqslant \theta|x|^{-(n+p s)} \text { for any } x \in \mathbb{R}^{n} \backslash\{0\} \text {, }
$$

hold true. A model for $\mathcal{L}_{K}^{p}$ is given by the fractional $p$-Laplacian $(-\Delta)_{p}^{s}$ which (up to normalization factors) may be defined for any $x \in \mathbb{R}^{n}$ as

$$
(-\Delta)_{p}^{s} u(x)=2 \lim _{\varepsilon \searrow 0} \int_{\mathbb{R}^{n} \backslash B_{\varepsilon}(x)} \frac{|u(x)-u(y)|^{p-2}(u(x)-u(y))}{|x-y|^{n+s p}} d x d y .
$$

For problem (1.12) the appropriate functional space where finding solution is $X_{0}^{s, p}(\Omega)$, defined as

$$
X_{0}^{s, p}(\Omega)=\left\{g \in X^{s, p}(\Omega): g=0 \text { a.e. in } \mathbb{R}^{n} \backslash \Omega\right\} .
$$

Here $X^{s, p}(\Omega)$ denotes the linear space of Lebesgue measurable functions $u: \mathbb{R}^{n} \rightarrow \mathbb{R}$ whose restrictions to $\Omega$ belong to $L^{p}(\Omega)$ and such that

$$
\text { the map }(x, y) \mapsto(u(x)-u(y))^{p} K(x-y) \text { is in } L^{1}(Q, d x d y) \text {, }
$$

where $Q=\mathbb{R}^{n} \times \mathbb{R}^{n} \backslash\left(\left(\mathbb{R}^{n} \backslash \Omega\right) \times\left(\mathbb{R}^{n} \backslash \Omega\right)\right)$. It is immediate to see that $X_{0}^{s, p}(\Omega)$ is a Banach space endowed with the following norm

$$
\|u\|_{s, p}=\left(\iint_{\mathbb{R}^{n} \times \mathbb{R}^{n}}|u(x)-u(y)|^{p} K(x-y) d x d y\right)^{1 / p} .
$$

When $p=2$ and $K(x)=|x|^{-(n+2 s)}$ the space $X_{0}^{s, p}(\Omega)$ coincides with $X_{0}^{s}(\Omega)$ defined in (2.2) (see [18, Lemma 5]). In such a case the statements of Theorem 1.1-Theorem 1.3 are still valid and their proofs can be performed exactly with the same arguments considered in the model case of the fractional Laplace operator $(-\Delta)^{s}$.

In order to treat problem (1.12) when $p \neq 2$ we have to adapt in a suitable way the arguments used for studying (1.1). Indeed, in this case the main difficulty is related to the fact that we have to understand how to decompose the space $X_{0}^{s, p}(\Omega)$. Indeed, when $p \neq 2$ the full spectrum of $(-\Delta)_{p}^{s}$ and of $\mathcal{L}_{K}^{p}$ is still almost unknown, even if some important 
properties of the first eigenvalue and of the higher order (variational) eigenvalues have been established in [11, 12]. We would recall that in [5] the authors proposed a definition of quasieigenvalues for $(-\Delta)_{p}^{s}$ and using them considered a suitable decomposition of $X_{0}^{s, p}(\Omega)$ which turns out to be the known one for $p=2$.

The paper is organized as follows. In Section 2 we introduce the variational formulation of the problem under consideration. Section 3 is devoted to the proof of the compactness property for problem (1.1). In Section 4 we conclude the proofs of Theorem 1.1-Theorem 1.3.

\section{VARIATIONAL SETTING}

Problem (1.1) has a variational structure and the natural space where finding solutions is the homogeneous fractional Sobolev space $H_{0}^{s}(\Omega)$. In order to study (1.1) it is important to encode the "boundary condition" $u=0$ in $\mathbb{R}^{n} \backslash \Omega$ (which is different from the classical case of the Laplacian, where it is required $u=0$ on $\partial \Omega$ ) in the weak formulation, by considering also that the interaction between $\Omega$ and its complementary in $\mathbb{R}^{n}$ gives a positive contribution in the so-called Gagliardo norm given as

$$
\|u\|_{H^{s}\left(\mathbb{R}^{n}\right)}=\|u\|_{L^{2}\left(\mathbb{R}^{n}\right)}+\left(\iint_{\mathbb{R}^{n} \times \mathbb{R}^{n}} \frac{|u(x)-u(y)|^{2}}{|x-y|^{n+2 s}} d x d y\right)^{1 / 2} .
$$

The functional space that takes into account this boundary condition will be denoted by $X_{0}^{s}(\Omega)$ and it is defined as

$$
X_{0}^{s}(\Omega)=\left\{u \in H^{s}\left(\mathbb{R}^{n}\right): u=0 \text { a.e. in } \mathbb{R}^{n} \backslash \Omega\right\} .
$$

We refer to [18, 22, for a general definition of $X_{0}^{s}(\Omega)$ and its properties. We also would like to point out that, when $\partial \Omega$ is continuous, by [9, Theorem 6] the space $X_{0}^{s}(\Omega)$ can be seen as the closure of $C_{0}^{\infty}(\Omega)$ with respect to the norm (2.1). This last point will play a crucial role in the proof of the compactness condition for the energy functional related to (1.1).

In $X_{0}^{s}(\Omega)$ we can consider the following norm

$$
\|u\|_{X_{0}^{s}(\Omega)}=\left(\iint_{\mathbb{R}^{n} \times \mathbb{R}^{n}} \frac{|u(x)-u(y)|^{2}}{|x-y|^{n+2 s}} d x d y\right)^{1 / 2},
$$

which is equivalent to the usual one defined in (2.1) (see [18, Lemma 6]). We also recall that $\left(X_{0}^{s}(\Omega),\|\cdot\|_{X_{0}^{s}(\Omega)}\right)$ is a Hilbert space, with the scalar product defined as

$$
\langle u, v\rangle_{X_{0}^{s}(\Omega)}=\iint_{\mathbb{R}^{n} \times \mathbb{R}^{n}} \frac{(u(x)-u(y))(v(x)-v(y))}{|x-y|^{n+2 s}} d x d y .
$$

From now on, in order to simplify the notation, we will denote $\|\cdot\|_{X_{0}^{s}(\Omega)}$ and $\langle\cdot, \cdot\rangle_{X_{0}^{s}(\Omega)}$ by $\|\cdot\|$ and $\langle\cdot, \cdot\rangle$ respectively, and $\|\cdot\|_{L^{q}(\Omega)}$ by $\|\cdot\|_{q}$ for any $q \in[1,+\infty]$.

A function $u \in X_{0}^{s}(\Omega)$ is said to be a (weak) solution of problem (1.1) if $u$ satisfies the following weak formulation

$$
\langle u, \varphi\rangle=\gamma \int_{\Omega}|u(x)|^{2^{*}-2} u(x) \varphi(x) d x+\int_{\Omega} f(x, u(x)) \varphi(x) d x,
$$

for any $\varphi \in X_{0}^{s}(\Omega)$. We observe that (2.5) represents the Euler-Lagrange equation of the functional $\mathcal{J}_{\gamma}: X_{0}^{s}(\Omega) \rightarrow \mathbb{R}$ defined as

$$
\mathcal{J}_{\gamma}(u)=\frac{1}{2}\|u\|^{2}-\frac{\gamma}{2^{*}}\|u\|_{2^{*}}^{2^{*}}-\int_{\Omega} F(x, u(x)) d x,
$$

where $F$ is as in (1.5). It is easily seen that $\mathcal{J}_{\gamma}$ is well defined thanks to (1.3)-(1.6) and [18, Lemma 6]. Moreover, $\mathcal{J}_{\gamma} \in C^{1}\left(X_{0}^{s}(\Omega)\right)$, thus critical points of $\mathcal{J}_{\gamma}$ are solutions to problem (2.5), that is weak solutions for (1.1).

The proofs of Theorem 1.1 and Theorem 1.2 are mainly based on variational and topological methods. Precisely, here we will perform the following version of the symmetric Mountain Pass Theorem (see [2, 4, 23]). 
Theorem 2.1 (Abstract critical point theorem). Let $E=V \oplus X$, where $E$ is a real Banach space and $V$ is finite dimensional. Suppose that $\mathcal{I} \in C^{1}(E, \mathbb{R})$ is a functional satisfying the following conditions:

$\left(I_{1}\right) \mathcal{I}(u)=\mathcal{I}(-u)$ and $\mathcal{I}(0)=0$;

$\left(I_{2}\right)$ there exists a constant $\rho>0$ such that $\left.\mathcal{I}\right|_{\partial B_{\rho} \cap X} \geqslant 0$;

$\left(I_{3}\right)$ there exists a subspace $W \subset E$ with $\operatorname{dim} V<\operatorname{dim} W<+\infty$ and there is $M>0$ such that $\max _{u \in W} \mathcal{I}(u)<M$;

$\left(I_{4}\right)$ considering $M>0$ from $\left(I_{3}\right), \mathcal{I}(u)$ satisfies $(P S)_{c}$ condition for $0 \leqslant c \leqslant M$.

Then, there exist at least $\operatorname{dim} W-\operatorname{codim} V$ pairs of non-trivial critical points of $\mathcal{I}$.

In order to prove our main results, the idea consists in applying Theorem 2.1 to the functional $\mathcal{J}_{\gamma}$. At this purpose note that when $f$ is odd in $t, \mathcal{J}_{\gamma}$ is even and also $\mathcal{J}_{\gamma}(0)=0$. Thus, condition $\left(I_{1}\right)$ of Theorem 2.1 is always verified by $\mathcal{J}_{\gamma}$ and we will not recall it in the sequel.

For the proof of Theorem 1.3 we will use the following version of the Mountain Pass Theorem (see [23]):

Theorem 2.2. Let $E$ be a real Banach space. Suppose that $\mathcal{I} \in C^{1}(E, \mathbb{R})$ is a functional satisfying the following conditions:

$\left(I_{1}\right) \mathcal{I}(0)=0$;

$\left(I_{2}\right)$ there exists a constant $\rho>0$ such that $\left.\mathcal{I}\right|_{\partial B_{\rho}} \geqslant 0$;

$\widehat{\left(I_{3}\right)}$ there exist $v_{1} \in \partial B_{1}$ and $M>0$ such that $\sup _{t \geqslant 0} \mathcal{I}\left(t v_{1}\right) \leqslant M$;

$\left(I_{4}\right)$ considering $M>0$ from $\left(I_{3}\right), \mathcal{I}(u)$ satisfies $(P S)_{c}$ condition for $0 \leqslant c \leqslant M$.

Then, $\mathcal{I}$ possesses a non-trivial critical point.

\section{The Palais-Smale Condition}

In this section we verify that the functional $\mathcal{J}_{\gamma}$ satisfies the $(P S)_{c}$ condition under a suitable level. For this, we use some preliminary estimates concerning the nonlinearity $f$ and its primitive $F$. By (1.3) and (1.6) for any $\varepsilon>0$ there exists a constant $C_{\varepsilon}>0$ such that

$$
|f(x, t) t| \leqslant C_{\varepsilon}+\varepsilon|t|^{2^{*}} \quad \text { for any } t \in \mathbb{R} \text { and a.e. } x \in \Omega
$$

and

$$
|F(x, t)| \leqslant C_{\varepsilon}+\frac{\varepsilon}{2^{*}}|t|^{2^{*}} \quad \text { for any } t \in \mathbb{R} \text { and a.e. } x \in \Omega .
$$

We recall that $\left\{u_{j}\right\}_{j \in \mathbb{N}} \subset X_{0}^{s}(\Omega)$ is a Palais-Smale sequence for $\mathcal{J}_{\gamma}$ at level $c \in \mathbb{R}$ (in short $(P S)_{c}$ sequence) if

$$
\mathcal{J}_{\gamma}\left(u_{j}\right) \rightarrow c \quad \text { and } \quad \mathcal{J}_{\gamma}^{\prime}\left(u_{j}\right) \rightarrow 0 \quad \text { as } j \rightarrow+\infty .
$$

We say that $\mathcal{J}_{\gamma}$ satisfies the Palais-Smale condition at level $c$ if any Palais-Smale sequence $\left\{u_{j}\right\}_{j \in \mathbb{N}}$ at level $c$ admits a convergent subsequence in $X_{0}^{s}(\Omega)$.

As usual, we first prove the boundedness of the $(P S)_{c}$ sequence.

Lemma 3.1. Let $f$ satisfy (1.3), (1.6) and (1.7). For any $\gamma>0$, let $c>0$ and $\left\{u_{j}\right\}_{j \in \mathbb{N}}$ be $a(P S)_{c}$ sequence for $\mathcal{J}_{\gamma}$.

Then, $\left\{u_{j}\right\}_{j \in \mathbb{N}}$ is bounded in $X_{0}^{s}(\Omega)$.

Proof. Fix $\gamma>0$. By (3.3) there exists $C>0$ such that

$$
\left|\mathcal{J}_{\gamma}\left(u_{j}\right)\right| \leqslant C \quad \text { and } \quad\left|\mathcal{J}_{\gamma}^{\prime}\left(u_{j}\right)\left(\frac{u_{j}}{\left\|u_{j}\right\|}\right)\right| \leqslant C \quad \text { for any } j \in \mathbb{N} \text {. }
$$


Moreover, by (1.7) and Hölder inequality we have

$$
\mathcal{J}_{\gamma}\left(u_{j}\right)-\frac{1}{2} \mathcal{J}_{\gamma}^{\prime}\left(u_{j}\right)\left(u_{j}\right) \geqslant \frac{s \gamma}{n}\left\|u_{j}\right\|_{2^{*}}^{2^{*}}-a_{1}|\Omega|-a_{2}|\Omega|^{\frac{2^{*}-\sigma}{2^{*}}}\left\|u_{j}\right\|_{2^{*}}^{\sigma} .
$$

From Young's inequality with exponents $p=2^{*} / \sigma$ and $q=2^{*} /\left(2^{*}-\sigma\right)$ we also get

$$
\left\|u_{j}\right\|_{2^{*}}^{\sigma} \leqslant \delta\left\|u_{j}\right\|_{2^{*}}^{2^{*}}+C_{\delta}
$$

for suitable $\delta, C_{\delta}>0$. The last inequality combined with (3.4) and (3.5) says that

$$
\left\|u_{j}\right\|_{2^{*}}^{2^{*}} \leqslant C^{\prime}\left(\left\|u_{j}\right\|+1\right)
$$

for another positive constant $C^{\prime}$.

Now, by (3.2), (3.4) and (3.6) we obtain

$$
C \geqslant \mathcal{J}_{\gamma}\left(u_{j}\right) \geqslant \frac{1}{2}\left\|u_{j}\right\|^{2}-\left(\frac{C^{\prime} \gamma}{2^{*}}-\frac{C^{\prime} \varepsilon}{2^{*}}\right)\left(1+\left\|u_{j}\right\|\right)-C_{\varepsilon}|\Omega|,
$$

which gives the boundedness of $\left\{u_{j}\right\}_{j \in \mathbb{N}}$ in $X_{0}^{s}(\Omega)$.

Now, we can prove the relatively compactness of a $(P S)_{c}$ sequence under a suitable level. Here, we must pay attention to the lack of compactness at level $L^{2^{*}}(\Omega)$.

Lemma 3.2. Let $f$ satisfy (1.3), (1.6) and (1.7).

Then, for any $M>0$ there exists $\gamma^{*}>0$ such that $\mathcal{J}_{\gamma}$ satisfies the $(P S)_{c}$ condition for any $c \leqslant M$, provided $0<\gamma<\gamma^{*}$.

Proof. Fix $M>0$. We set

$$
\gamma^{*}=\min \left\{S(n, s),\left[(S(n, s))^{\frac{n}{2 s}}\left(\frac{s}{n(M+A)}\right)^{\frac{2^{*}}{2^{*}-\sigma}}\right]^{\frac{1}{n / 2 s-2^{*} /\left(2^{*}-\sigma\right)}}\right\}
$$

with

$$
A=a_{1}|\Omega|+a_{2}|\Omega|^{\frac{2^{*}-\sigma}{2^{*}}},
$$

where $a_{1}, a_{2}, \sigma$ are the constants given in (1.7), while $S(n, s)$ is the best constant of the fractional Sobolev embedding (see [18, Lemma 6]) defined as

$$
S(n, s)=\inf _{v \in H^{s}\left(\mathbb{R}^{n}\right) \backslash\{0\}} \frac{\iint_{\mathbb{R}^{n} \times \mathbb{R}^{n}} \frac{|v(x)-v(y)|^{2}}{|x-y|^{n+2 s}} d x d y}{\left(\int_{\mathbb{R}^{n}}|v(x)|^{2^{*}}\right)^{2 / 2^{*}}}>0 .
$$

Given $\gamma<\gamma^{*}$ and $c<M$, let us consider a $(P S)_{c}$ sequence $\left\{u_{j}\right\}_{j \in \mathbb{N}}$ for $\mathcal{J}_{\gamma}$. Since by Lemma 3.1] we have that $\left\{u_{j}\right\}_{j \in \mathbb{N}}$ is bounded in $X_{0}^{s}(\Omega)$, by applying also [17, Lemma 8] and [6. Theorem IV.9], there exists $u \in X_{0}^{s}(\Omega)$ such that, up to a subsequence,

$$
\begin{gathered}
u_{j} \rightarrow u \quad \text { weakly in } X_{0}^{s}(\Omega), \\
u_{j} \rightarrow u \quad \text { in } L^{q}(\Omega),
\end{gathered}
$$

with $q \in\left[1,2^{*}\right)$ and

$$
u_{j} \rightarrow u \quad \text { a.e in } \Omega \text {, }
$$

as $j \rightarrow+\infty$.

Now, we claim that

$$
\left\|u_{j}\right\|^{2} \rightarrow\|u\|^{2} \quad \text { as } j \rightarrow+\infty
$$

which easily implies that $u_{j} \rightarrow u$ in $X_{0}^{s}(\Omega)$ as $j \rightarrow+\infty$, thanks to (3.10). 
First of all, from Phrokorov's Theorem we deduce the existence of two positive measures $\mu$ and $\nu$ on $\mathbb{R}^{n}$ such that

$$
\left|(-\Delta)^{s / 2} u_{j}(x)\right|^{2} d x \stackrel{*}{\rightarrow} \mu \quad \text { and } \quad\left|u_{j}(x)\right|^{2^{*}} d x \rightarrow \nu \quad \text { in } \mathcal{M}\left(\mathbb{R}^{n}\right)
$$

as $j \rightarrow+\infty$. By [9, Theorem 6], thanks to our assumptions on $\partial \Omega$, it is easy to see that $X_{0}^{s}(\Omega)$ can also be defined as the closure of $C_{0}^{\infty}(\Omega)$ with respect to the norm (2.1). Hence, $X_{0}^{s}(\Omega)$ is consistent with the functional space introduced in [14. Thus, by [14, Theorem 2] we obtain an at most countable set of distinct points $\left\{x_{i}\right\}_{i \in J}$, non-negative numbers $\left\{\nu_{i}\right\}_{i \in J}$, $\left\{\mu_{i}\right\}_{i \in J}$ and a positive measure $\widetilde{\mu}$, with $\operatorname{Supp} \widetilde{\mu} \subset \bar{\Omega}$, such that

$$
\nu=|u(x)|^{2^{*}} d x+\sum_{i \in J} \nu_{i} \delta_{x_{i}}, \quad \mu=\left|(-\Delta)^{s / 2} u(x)\right|^{2} d x+\widetilde{\mu}+\sum_{i \in J} \mu_{i} \delta_{x_{i}},
$$

and

$$
\nu_{i} \leqslant S(n, s)^{-\frac{2^{*}}{2}} \mu_{i}^{\frac{2^{*}}{2}}
$$

for any $i \in J$, where $S(n, s)$ is the constant given in (3.9). Now, in order to prove (3.13) we proceed by steps.

Step 1. Fix $i_{0} \in J$. Then, either $\nu_{i_{0}}=0$ or

$$
\nu_{i_{0}} \geqslant\left[\frac{S(n, s)}{\gamma}\right]^{n / 2 s} .
$$

Proof. Let $\psi \in C_{0}^{\infty}\left(\mathbb{R}^{n},[0,1]\right)$ be such that $\psi \equiv 1$ in $B(0,1)$ and $\psi \equiv 0$ in $\mathbb{R}^{n} \backslash B(0,2)$. For any $\delta \in(0,1)$ we set

$$
\psi_{\delta, i_{0}}(x)=\psi\left(\left(x-x_{i_{0}}\right) / \delta\right) .
$$

Clearly the sequence $\left\{\psi_{\delta, i_{0}} u_{j}\right\}_{j \in \mathbb{N}}$ is bounded in $X_{0}^{s}(\Omega)$ by Lemma 3.1. and so by (3.3) it follows that

$$
\mathcal{J}_{\gamma}^{\prime}\left(u_{j}\right)\left(\psi_{\delta, i_{0}} u_{j}\right) \rightarrow 0
$$

as $j \rightarrow+\infty$. In other words

$$
\begin{aligned}
& o(1)+\iint_{\mathbb{R}^{n} \times \mathbb{R}^{n}} \frac{\left(u_{j}(x)-u_{j}(y)\right)\left(\psi_{\delta, i_{0}}(x) u_{j}(x)-\psi_{\delta, i_{0}}(y) u_{j}(y)\right)}{|x-y|^{n+2 s}} d x d y \\
& \quad=\gamma \int_{\Omega}\left|u_{j}(x)\right|^{2^{*}} \psi_{\delta, i_{0}}(x) d x+\int_{\Omega} f\left(x, u_{j}(x)\right) \psi_{\delta, i_{0}}(x) u_{j}(x) d x
\end{aligned}
$$

as $j \rightarrow+\infty$.

By [7, Proposition 3.6] and taking into account the definition of $(-\Delta)^{s}$ given in (1.2), we know that for any $v \in X_{0}^{s}(\Omega)$

$$
\iint_{\mathbb{R}^{n} \times \mathbb{R}^{n}} \frac{|v(x)-v(y)|^{2}}{|x-y|^{n+2 s}} d x d y=\int_{\mathbb{R}^{n}}\left|(-\Delta)^{s / 2} v(x)\right|^{2} d x .
$$

By taking derivative of the above equality, for any $v, w \in X_{0}^{s}(\Omega)$ we obtain

$$
\iint_{\mathbb{R}^{n} \times \mathbb{R}^{n}} \frac{(v(x)-v(y))(w(x)-w(y))}{|x-y|^{n+2 s}} d x d y=\int_{\mathbb{R}^{n}}(-\Delta)^{s / 2} v(x)(-\Delta)^{s / 2} w(x) d x .
$$

Furthermore, for any $v, w \in X_{0}^{s}(\Omega)$ we have

$$
(-\Delta)^{s / 2}(v w)=v(-\Delta)^{s / 2} w+w(-\Delta)^{s / 2} v-2 I_{s / 2}(v, w),
$$

where the last term is defined, in the principal value sense, as follows

$$
I_{s / 2}(v, w)(x)=P . V \cdot \int_{\mathbb{R}^{n}} \frac{(v(x)-v(y))(w(x)-w(y))}{|x-y|^{n+s}} d y
$$

for any $x \in \mathbb{R}^{n}$. 
Thus, by (3.19) and (3.20) the integral in the left-hand side of (3.18) becomes

$$
\begin{aligned}
& \iint_{\mathbb{R}^{n} \times \mathbb{R}^{n}} \frac{\left(u_{j}(x)-u_{j}(y)\right)\left(\psi_{\delta, i_{0}}(x) u_{j}(x)-\psi_{\delta, i_{0}}(y) u_{j}(y)\right)}{|x-y|^{n+2 s}} d x d y \\
& =\int_{\mathbb{R}^{n}}(-\Delta)^{s / 2} u_{j}(x)(-\Delta)^{s / 2}\left(\psi_{\delta, i_{0}} u_{j}\right)(x) d x \\
& =\int_{\mathbb{R}^{n}} u_{j}(x)(-\Delta)^{s / 2} u_{j}(x)(-\Delta)^{s / 2} \psi_{\delta, i_{0}}(x) d x \\
& \quad+\int_{\mathbb{R}^{n}}\left|(-\Delta)^{s / 2} u_{j}(x)\right|^{2} \psi_{\delta, i_{0}}(x) d x \\
& \quad-2 \int_{\mathbb{R}^{n}}(-\Delta)^{s / 2} u_{j}(x) \int_{\mathbb{R}^{n}} \frac{\left(u_{j}(x)-u_{j}(y)\right)\left(\psi_{\delta, i_{0}}(x)-\psi_{\delta, i_{0}}(y)\right)}{|x-y|^{n+s}} d x d y .
\end{aligned}
$$

By [3, Lemma 2.8 and Lemma 2.9] we have

$$
\lim _{\delta \rightarrow 0} \lim _{j \rightarrow+\infty}\left|\int_{\mathbb{R}^{n}} u_{j}(x)(-\Delta)^{s / 2} u_{j}(x)(-\Delta)^{s / 2} \psi_{\delta, i_{0}}(x) d x\right|=0
$$

and

$$
\lim _{\delta \rightarrow 0} \lim _{j \rightarrow+\infty}\left|\int_{\mathbb{R}^{n}}(-\Delta)^{s / 2} u_{j}(x) \int_{\mathbb{R}^{n}} \frac{\left(u_{j}(x)-u_{j}(y)\right)\left(\psi_{\delta, i_{0}}(x)-\psi_{\delta, i_{0}}(y)\right)}{|x-y|^{n+s}} d x d y\right|=0 .
$$

Then, by combining (3.21)-(3.23) and (3.14) $-(3.15)$ we get

$$
\lim _{\delta \rightarrow 0} \lim _{j \rightarrow+\infty} \iint_{\mathbb{R}^{n} \times \mathbb{R}^{n}} \frac{\left(u_{j}(x)-u_{j}(y)\right)\left(\psi_{\delta, i_{0}}(x) u_{j}(x)-\psi_{\delta, i_{0}}(y) u_{j}(y)\right)}{|x-y|^{n+2 s}} d x d y \geqslant \mu_{i_{0}} .
$$

While, by (3.1) and the Dominated Convergence Theorem we get

$$
\int_{B\left(x_{i_{0}}, 2 \delta\right)} f\left(x, u_{j}(x)\right) u_{j}(x) \psi_{\delta, i_{0}}(x) d x \rightarrow \int_{B\left(x_{i_{0}}, 2 \delta\right)} f(x, u(x)) u(x) \psi_{\delta, i_{0}}(x) d x \quad \text { as } j \rightarrow+\infty
$$

and so by sending $\delta \rightarrow 0$ we observe that

$$
\lim _{\delta \rightarrow 0} \lim _{j \rightarrow+\infty} \int_{B\left(x_{i_{0}}, 2 \delta\right)} f\left(x, u_{j}(x)\right) u_{j}(x) \psi_{\delta, i_{0}}(x) d x=0 .
$$

Furthermore, by (3.14) it follows that

$$
\int_{\Omega}\left|u_{j}(x)\right|^{2^{*}} \psi_{\delta, i_{0}}(x) d x \rightarrow \int_{\Omega} \psi_{\delta, i_{0}}(x) d \nu \quad \text { as } j \rightarrow+\infty .
$$

Finally, by combining this last formula with (3.18), (3.24) and (3.25) we get

$$
\nu_{i_{0}} \geqslant \frac{\mu_{i_{0}}}{\gamma}
$$

Thus, from this and (3.16) with $i=i_{0}$ we have that

$$
\nu_{i_{0}} \geqslant \frac{\nu_{i_{0}}^{2 / 2^{*}} S(n, s)}{\gamma},
$$

which yields that either $\nu_{i_{0}}=0$ or $\nu_{i_{0}}$ verifies (3.17). This ends the proof of Step 1.

Step 2. Estimate (3.17) can not occur, hence $\nu_{i_{0}}=0$.

Proof. For this, it is enough to see that

$$
\int_{\Omega} d \nu<\left[\frac{S(n, s)}{\gamma}\right]^{\frac{n}{2 s}} .
$$

For this, let us consider two cases. First of all, assume that

$$
\int_{\Omega} d \nu \leqslant 1
$$


Since $\gamma<\gamma^{*}$ and by (3.7) (which implies that $\gamma^{*}<S(n, s)$ ) we have

$$
1<\left(\frac{S(n, s)}{\gamma}\right)^{\frac{n}{2 s}}
$$

from which immediately follows (3.26), thanks to (3.27).

Now, assume that $\int_{\Omega} d \nu>1$. Since $\left\{u_{j}\right\}_{j \in \mathbb{N}}$ is a $(P S)_{c}$ sequence for $\mathcal{J}_{\gamma}$, arguing as in Lemma 3.1 (see formula (3.5) ) we get

$$
\mathcal{J}_{\gamma}\left(u_{j}\right)-\frac{1}{2} \mathcal{J}_{\gamma}^{\prime}\left(u_{j}\right)\left(u_{j}\right) \geqslant \frac{s \gamma}{n}\left\|u_{j}\right\|_{2^{*}}^{2^{*}}-a_{1}|\Omega|-a_{2}|\Omega|^{\frac{2^{*}-\sigma}{2^{*}}}\left\|u_{j}\right\|_{2^{*}}^{\sigma} .
$$

By sending $j \rightarrow+\infty$ in (3.28) and using (3.3), (3.14) we obtain

$$
\begin{aligned}
\frac{s \gamma}{n} \int_{\Omega} d \nu & \leqslant c+a_{1}|\Omega|+a_{2}|\Omega|^{\frac{2^{*}-\sigma}{2^{*}}}\left(\int_{\Omega} d \nu\right)^{\frac{\sigma}{2^{*}}} \\
& \leqslant\left(M+a_{1}|\Omega|+a_{2}|\Omega|^{\frac{2^{*}-\sigma}{2^{*}}}\right)\left(\int_{\Omega} d \nu\right)^{\frac{\sigma}{2^{*}}} \\
& =(M+A)\left(\int_{\Omega} d \nu\right)^{\frac{\sigma}{2^{*}}},
\end{aligned}
$$

thanks to the choice of $c \leqslant M$ and the definition of $A$ given in (3.8). Hence we get

$$
\int_{\Omega} d \nu \leqslant\left[\frac{n(M+A)}{s \gamma}\right]^{\frac{2^{*}}{2^{*}-\sigma}} .
$$

By (3.7) and the fact that $\gamma<\gamma^{*}$ we know that

$$
\gamma<\left[(S(n, s))^{\frac{n}{2 s}}\left(\frac{s}{n(M+A)}\right)^{\frac{2^{*}}{2^{*}-\sigma}}\right]^{\frac{1}{n / 2 s-2^{*} /\left(2^{*}-\sigma\right)}},
$$

that is

which yields

$$
\gamma^{\frac{n}{2 s}-\frac{2^{*}}{2^{*}-\sigma}}<(S(n, s))^{\frac{n}{2 s}}\left(\frac{s}{n(M+A)}\right)^{\frac{2^{*}}{2^{*}-\sigma}},
$$

$$
\left[\frac{n(M+A)}{s \gamma}\right]^{\frac{2^{*}}{2^{*}-\sigma}}<\left(\frac{S(n, s)}{\gamma}\right)^{\frac{n}{2 s}} .
$$

From this and (3.29) we get (3.26). Thus, the proof of Step 2 is complete and $\nu_{i_{0}}=0$.

Step 3. Claim (3.13) holds true.

Proof. By considering that $i_{0}$ was arbitrary in Step 1 we deduce that $\nu_{i}=0$ for any $i \in J$. As a consequence, also from (3.14) and (3.15) it follows that $u_{j} \rightarrow u$ in $L^{2^{*}}(\Omega)$ as $j \rightarrow+\infty$. Thus, by (3.1), the fact that

$$
\mathcal{J}_{\gamma}^{\prime}\left(u_{j}\right) \rightarrow 0 \quad \text { as } j \rightarrow+\infty
$$

(being $\left\{u_{j}\right\}_{j \in \mathbb{N}}$ a $(P S)_{c}$ sequence for $\left.\mathcal{J}_{\gamma}\right)$ and the Dominated Convergence Theorem, we have

$$
\lim _{j \rightarrow+\infty}\left\|u_{j}\right\|^{2}=\gamma \int_{\Omega}|u(x)|^{2^{*}} d x+\int_{\Omega} f(x, u(x)) u(x) d x .
$$

Moreover, by remembering that $u_{j} \rightarrow u$ in $X_{0}^{s}(\Omega)$ and by using again (3.1), (3.30) and the Dominated Convergence Theorem, we have

$$
\langle u, \varphi\rangle=\gamma \int_{\Omega}|u(x)|^{2^{*}-2} u(x) \varphi(x) d x+\int_{\Omega} f(x, u(x)) \varphi(x) d x,
$$

for any $\varphi \in X_{0}^{s}(\Omega)$. Thus, by combining (3.31) and (3.32) with $\varphi=u$ we get the claim (3.13), concluding the proof of Step 3 . 
Hence, the proof of Lemma 3.2 is complete.

\section{MAIN THEOREMS}

This section is devoted to the proof of the main results of the paper. In particular here we study the geometry of the functional $\mathcal{J}_{\gamma}$.

At first, we need some notation. In what follows $\left\{\lambda_{j}\right\}_{j \in \mathbb{N}}$ denotes the sequence of the eigenvalues of the following problem

$$
\begin{cases}(-\Delta)^{s} u=\lambda u & \text { in } \Omega \\ u=0 & \text { in } \mathbb{R}^{n} \backslash \Omega,\end{cases}
$$

with

$$
\begin{gathered}
0<\lambda_{1}<\lambda_{2} \leqslant \ldots \leqslant \lambda_{j} \leqslant \lambda_{j+1} \leqslant \ldots \\
\lambda_{j} \rightarrow+\infty \text { as } j \rightarrow+\infty,
\end{gathered}
$$

and with $e_{j}$ as eigenfunction corresponding to $\lambda_{j}$. Also, we choose $\left\{e_{j}\right\}_{j \in \mathbb{N}}$ normalized in such a way that this sequence provides an orthonormal basis of $L^{2}(\Omega)$ and an orthogonal basis of $X_{0}^{s}(\Omega)$. For a complete study of the spectrum of the fractional Laplace operator $(-\Delta)^{s}$ we refer to [16, Proposition 2.3], [19, Proposition 9 and Appendix A] and [20, Proposition 4].

Along the paper, for any $j \in \mathbb{N}$ we also set

$$
\mathbb{P}_{j+1}=\left\{u \in X_{0}^{s}(\Omega):\left\langle u, e_{i}\right\rangle=0 \quad \text { for any } i=1, \ldots, j\right\} \quad\left(\text { with } \mathbb{P}_{1}=X_{0}^{s}(\Omega)\right),
$$

as defined also in [19, Proposition 9 and Appendix A], while

$$
\mathbb{H}_{j}=\operatorname{span}\left\{e_{1}, \ldots, e_{j}\right\}
$$

will denote the linear subspace generated by the first $j$ eigenfunctions of $(-\Delta)^{s}$. It is immediate to observe that $\mathbb{P}_{j+1}=\mathbb{H}_{j}^{\perp}$ with respect to the scalar product in $X_{0}^{s}(\Omega)$ defined as in formula (2.4). Thus, since $X_{0}^{s}(\Omega)$ is a Hilbert space (see [18, Lemma 7] and (2.4)), we can write it as a direct sum as follows

$$
X_{0}^{s}(\Omega)=\mathbb{H}_{j} \oplus \mathbb{P}_{j+1}
$$

for any $j \in \mathbb{N}$. Moreover, since $\left\{e_{j}\right\}_{j \in \mathbb{N}}$ is an orthogonal basis of $X_{0}^{s}(\Omega)$, it is easy to see that for any $j \in \mathbb{N}$

$$
\mathbb{P}_{j+1}=\overline{\operatorname{span}\left\{e_{i}: i \geqslant j+1\right\}} .
$$

Now, before studying and proving the geometric features for $\mathcal{J}_{\gamma}$ we need a stronger version of the classical Sobolev embedding. Here the constant of the embedding can be chosen and controlled a priori.

Lemma 4.1. Let $r \in\left[2,2^{*}\right)$ and $\delta>0$.

Then, there exists $j \in \mathbb{N}$ such that $\|u\|_{r}^{r} \leqslant \delta\|u\|^{r}$ for any $u \in \mathbb{P}_{j+1}$.

Proof. By contradiction, we suppose that there exists $\delta>0$ such that for any $j \in \mathbb{N}$ there exists $u_{j} \in \mathbb{P}_{j+1}$ which verifies $\left\|u_{j}\right\|_{r}^{r}>\delta\left\|u_{j}\right\|^{r}$. Considering $v_{j}=u_{j} /\left\|u_{j}\right\|_{r}$, we have that $v_{j} \in \mathbb{P}_{j+1}$

$$
\left\|v_{j}\right\|_{r}=1
$$

and $\left\|v_{j}\right\|<1 / \delta$ for any $j \in \mathbb{N}$. Thus, the sequence $\left\{v_{j}\right\}_{j \in \mathbb{N}}$ is bounded in $X_{0}^{s}(\Omega)$ and we may suppose that there exists $v \in X_{0}^{s}(\Omega)$ such that, up to a subsequence,

$$
v_{j} \rightarrow v \quad \text { in } \quad X_{0}^{s}(\Omega)
$$

and

$$
v_{j} \rightarrow v \quad \text { in } L^{r}(\Omega)
$$


as $j \rightarrow+\infty$. Hence, by (4.3) and (4.4) we deduce that

$$
\|v\|_{r}=1 \text {. }
$$

Moreover, since $\left\{e_{j}\right\}_{j \in \mathbb{N}}$ is an orthogonal basis of $X_{0}^{s}(\Omega)$ by [19, Proposition 9], we can write $v$ as follows

$$
v=\sum_{j=1}^{\infty}\left\langle v, e_{j}\right\rangle e_{j} .
$$

Now, given $k \in \mathbb{N}$ we have $\left\langle v_{j}, e_{k}\right\rangle=0$ for any $j \geqslant k$, since $v_{j} \in \mathbb{P}_{j+1}$. From this we deduce that $\left\langle v, e_{k}\right\rangle=0$ for any $k \in \mathbb{N}$, which clearly implies that $v \equiv 0$. On the other hand, this contradicts (4.5). Hence, Lemma 4.1] holds true.

4.1. Geometric setting for Theorem 1.1. In order to prove Theorem 1.1, we just have to verify that the energy functional $\mathcal{J}_{\gamma}$ satisfies $\left(I_{2}\right)$ and $\left(I_{3}\right)$ of Theorem 2.1. For this we will consider $V=\mathbb{H}_{j}$ and $X=\mathbb{P}_{j+1}$, with $j \in \mathbb{N}$ chosen as in the following result:

Lemma 4.2. Let $f$ satisfy (1.8).

Then, there exist $\widetilde{\gamma}>0, j \in \mathbb{N}$ and $\rho, \alpha>0$ such that $\mathcal{J}_{\gamma}(u) \geqslant \alpha$, for any $u \in \mathbb{P}_{j+1}$ with $\|u\|=\rho$, and $0<\gamma<\widetilde{\gamma}$.

Proof. Take $\gamma>0$. By (1.8) and [18, Lemma 6] we get a suitable constant $c>0$ such that

$$
\mathcal{J}_{\gamma}(u) \geqslant \frac{1}{2}\|u\|^{2}-b_{1}\|u\|_{\theta}^{\theta}-b_{2}|\Omega|-\gamma c\|u\|^{2^{*}},
$$

for any $u \in X_{0}^{s}(\Omega)$. Let $\delta>0$ : we will fix it in the sequel. By (4.6) and Lemma 4.1 there exists $j \in \mathbb{N}$ such that

$$
\mathcal{J}_{\gamma}(u) \geqslant\|u\|^{2}\left(\frac{1}{2}-b_{1} \delta\|u\|^{\theta-2}\right)-b_{2}|\Omega|-\gamma c\|u\|^{2^{*}},
$$

for any $u \in \mathbb{P}_{j+1}$.

Now, consider $\|u\|=\rho=\rho(\delta)$, with $\rho$ such that $b_{1} \delta \rho^{\theta-2}=1 / 4$, so that

$$
\mathcal{J}_{\gamma}(u) \geqslant \frac{1}{4} \rho^{2}-b_{2}|\Omega|-\gamma c \rho^{2^{*}}
$$

for any $u \in \mathbb{P}_{j+1}$, thanks to (4.7).

Now, observe that $\rho(\delta) \rightarrow+\infty$ as $\delta \rightarrow 0$, since $\theta>2$. Hence, we can choose $\delta$ sufficiently small such that $\rho^{2} / 4-b_{2}|\Omega| \geqslant \rho^{2} / 8$, which yields

$$
\mathcal{J}_{\gamma}(u) \geqslant \frac{1}{8} \rho^{2}-\gamma c \rho^{2^{*}}
$$

for any $u \in \mathbb{P}_{j+1}$ with $\|u\|=\rho$.

Finally, let $\widetilde{\gamma}>0$ be such that $\frac{1}{8} \rho^{2}-\widetilde{\gamma} c \rho^{2^{*}}=\alpha>0$. Then we get

$$
\mathcal{J}_{\gamma}(u) \geqslant \mathcal{J}_{\widetilde{\gamma}}(u) \geqslant \alpha
$$

for any $u \in \mathbb{P}_{j+1}$ with $\|u\|=\rho$ and any $\gamma \in(0, \widetilde{\gamma})$, concluding the proof.

Lemma 4.3. Let $f$ satisfy (1.9) and let $l \in \mathbb{N}$.

Then, there exist a subspace $W$ of $X_{0}^{s}(\Omega)$ and a constant $M_{l}>0$, independent of $\gamma$, such that $\operatorname{dim} W=l$ and $\max _{u \in W} \mathcal{J}_{0}(u)<M_{l}$.

Proof. Here we can argue exactly as in [23, Lemma 4.3] where the classical case of the Laplacian was considered. For this, we can use also the properties of eigenfunctions of $(-\Delta)^{s}$ (see [19]). 
Proof of Theorem 1.1. By Lemma 4.2 we find $j \in \mathbb{N}$ and $\widetilde{\gamma}>0$ such that $\mathcal{J}_{\gamma}$ satisfies $\left(I_{2}\right)$ in $X=\mathbb{P}_{j+1}$, for any $0<\gamma<\widetilde{\gamma}$. While, by Lemma 4.3 for any $k \in \mathbb{N}$ there is a subspace $W \subset X_{0}^{s}(\Omega)$ with $\operatorname{dim} W=k+j$ and such that $\mathcal{J}_{\gamma}$ satisfies $\left(I_{3}\right)$ with $M=M_{j+k}>0$ for any $\gamma>0$, since $\mathcal{J}_{\gamma}<\mathcal{J}_{0}$.

Finally, we note that by Lemma 3.2, considering $\widetilde{\gamma}$ smaller if necessary, we have that $\mathcal{J}_{\gamma}$ satisfies $\left(I_{4}\right)$ for any $0<\gamma<\widetilde{\gamma}$. Thus, we may apply Theorem 2.1 to conclude that $\mathcal{J}_{\gamma}$ admits $k$ pairs of non-trivial critical points for $\gamma>0$ sufficiently small. Hence, Theorem 1.1 is proved.

4.2. Geometric setting for Theorem 1.2. We apply again Theorem 2.1 to the functional $\mathcal{J}_{\gamma}$. By considering $\lambda_{j} \leqslant \lambda_{k}$ as in (1.10) and (1.11), we have two cases. When $j=1$ we set $V=\{0\}$, so $X=X_{0}^{s}(\Omega)$ : note that this is consistent with the situation $\mathbb{P}_{1}=X_{0}^{s}(\Omega)$. While if $j>1$ we consider $X=\mathbb{P}_{j}$ and $V=\mathbb{H}_{j-1}$. Moreover, we set $W=\mathbb{H}_{k}$ as subspace of $X_{0}^{s}(\Omega)$ in $\left(I_{3}\right)$.

Now, in order to verify the geometric assumptions $\left(I_{2}\right)$ and $\left(I_{3}\right)$ in Theorem 2.1 we consider here two different characterizations of the eigenvalues of $(-\Delta)^{s}$. That is, for any $j \in \mathbb{N}$ by [19, Proposition 9] we have that

$$
\lambda_{j}=\min _{u \in \mathbb{P}_{j} \backslash\{0\}} \frac{\|u\|^{2}}{\|u\|_{2}^{2}},
$$

while from [16, Proposition 2.3] we know that

$$
\lambda_{j}=\max _{u \in \mathbb{H}_{j} \backslash\{0\}} \frac{\|u\|^{2}}{\|u\|_{2}^{2}} .
$$

Moreover, we need the following technical lemma:

Lemma 4.4. Let $a: \Omega \rightarrow \mathbb{R}$ be the measurable function given in (1.10). Then, there exists $\beta>0$ such that for any $u \in \mathbb{P}_{j}$

$$
\|u\|^{2}-\int_{\Omega} a(x)|u(x)|^{2} d x \geqslant \beta\|u\|_{2}^{2} .
$$

Proof. We argue by contradiction and we suppose that for any $i \in \mathbb{N}$ there exists $u_{i} \in \mathbb{P}_{j}$ such that

$$
\left\|u_{i}\right\|^{2}-\int_{\Omega} a(x)\left|u_{i}(x)\right|^{2} d x<\frac{1}{i}\left\|u_{i}\right\|_{2}^{2} .
$$

Let $v_{i}=u_{i} /\left\|u_{i}\right\|_{2}$. Of course, $v_{i} \in \mathbb{P}_{j}$ and

$$
\left\|v_{i}\right\|_{2}=1
$$

for any $i \in \mathbb{N}$. By (1.10), (4.8), (4.10) and (4.11) we get

$$
\begin{aligned}
\lambda_{j} & \leqslant\left\|v_{i}\right\|^{2} \\
& <\int_{\Omega} a(x)\left|v_{i}(x)\right|^{2} d x+\frac{1}{i} \\
& \leqslant \lambda_{j} \int_{\Omega}\left|v_{i}(x)\right|^{2} d x+\frac{1}{i} \\
& \leqslant \lambda_{j}+\frac{1}{i}
\end{aligned}
$$

for any $i \in \mathbb{N}$. From this, we have that $\left\{v_{i}\right\}_{i \in \mathbb{N}}$ is a bounded sequence in $X_{0}^{s}(\Omega)$. Therefore, by applying [18, Lemma 8] and [6, Theorem IV.9] there exists $v \in X_{0}^{s}(\Omega)$ such that, up to a subsequence, $v_{i}$ converges to $v$ weakly in $X_{0}^{s}(\Omega)$, strongly in $L^{2}(\Omega)$ and a.e. in $\Omega$ as $j \rightarrow+\infty$ and $\left|v_{i}\right| \leqslant h \in L^{2}(\Omega)$ a.e. in $\Omega$. Thus, by (4.11) we know that $\|v\|_{2}=1$, so that $v$ is almost everywhere different from zero in $\Omega$, i.e.

$$
v \not \equiv 0 \text { in } \Omega \text {. }
$$


By sending $i \rightarrow+\infty$ in (4.12) and using the Dominated Convergence Theorem and (4.10), we get

$$
\int_{\Omega}\left(\lambda_{j}-a(x)\right)|v(x)|^{2} d x=0 .
$$

Then, (1.10), (4.13) and (4.14) implies that

$$
a(x)=\lambda_{j} \quad \text { a.e. in } \Omega,
$$

which contradicts the assumption (1.10). Hence, Lemma 4.4 holds true.

Now we are ready to prove that $\mathcal{J}_{\gamma}$ satisfies $\left(I_{2}\right)$ and $\left(I_{3}\right)$ of Theorem 2.1 ,

Lemma 4.5. Let $f$ satisfy (1.3), (1.6) and (1.10).

Then, for any $\gamma>0$ there exist $\rho, \alpha>0$ such that $\mathcal{J}_{\gamma}(u) \geqslant \alpha$ for any $u \in \mathbb{P}_{j}$ with $\|u\|=\rho$.

Proof. Fix $\gamma>0$. By (1.3), (1.6) and (1.10), for any $\varepsilon>0$ there exists $C_{\varepsilon}>0$ such that

$$
|F(x, t)| \leqslant \frac{C_{\varepsilon}}{2^{*}}|t|^{2^{*}}+\frac{a(x)+\varepsilon}{2}|t|^{2},
$$

for any $t \in \mathbb{R}$ and a.e. $x \in \Omega$.

Now, let $\beta>0$ be as in Lemma 4.4 and $\varepsilon^{\prime}>0$ be such that $\beta-\varepsilon^{\prime} \lambda_{j}>0$. Thus, by (1.10) and Lemma 4.4, we have

$$
\begin{aligned}
\|u\|^{2}-\int_{\Omega} a(x)|u(x)|^{2} d x & =\frac{1+\varepsilon^{\prime}}{1+\varepsilon^{\prime}}\left(\|u\|^{2}-\int_{\Omega} a(x)|u(x)|^{2} d x\right) \\
& =\frac{\varepsilon^{\prime}}{1+\varepsilon^{\prime}}\|u\|^{2}+\frac{1}{1+\varepsilon^{\prime}}\left(\|u\|^{2}-\int_{\Omega} a(x)|u(x)|^{2} d x-\varepsilon^{\prime} \int_{\Omega} a(x)|u(x)|^{2} d x\right) \\
& \geqslant \frac{\varepsilon^{\prime}}{1+\varepsilon^{\prime}}\|u\|^{2}+\frac{1}{1+\varepsilon^{\prime}}\left(\beta\|u\|_{2}^{2}-\varepsilon^{\prime} \int_{\Omega} a(x)|u(x)|^{2} d x\right) \\
& \geqslant \frac{\varepsilon^{\prime}}{1+\varepsilon^{\prime}}\|u\|^{2}+\int_{\Omega}\left(\beta-\varepsilon^{\prime} \lambda_{j}\right)|u(x)|^{2} d x \\
& \geqslant \frac{\varepsilon^{\prime}}{1+\varepsilon^{\prime}}\|u\|^{2}
\end{aligned}
$$

for any $u \in \mathbb{P}_{j}$. From this and by (4.15) we get

$$
\begin{aligned}
\mathcal{J}_{\gamma}(u) & =\frac{1}{2}\|u\|^{2}-\frac{\gamma}{2^{*}}\|u\|_{2^{*}}^{2^{*}}-\int_{\Omega} F(x, u(x)) d x \\
& \geqslant \frac{1}{2}\left(\|u\|^{2}-\int_{\Omega} a(x)|u(x)|^{2} d x\right)-\frac{1}{2^{*}}\left(\gamma+C_{\varepsilon}\right)\|u\|_{2^{*}}^{2^{*}}-\frac{\varepsilon}{2}\|u\|_{2}^{2} \\
& \geqslant \frac{\varepsilon^{\prime}}{2\left(1+\varepsilon^{\prime}\right)}\|u\|^{2}-\frac{1}{2^{*}}\left(\gamma+C_{\varepsilon}\right)\|u\|_{2^{*}}^{2^{*}}-\frac{\varepsilon}{2}\|u\|_{2}^{2}
\end{aligned}
$$

for any $u \in \mathbb{P}_{j}$. Thus, by [18, Lemma 6] and taking $\varepsilon>0$ sufficiently small, there exist constants $K, C>0$ such that

$$
\mathcal{J}_{\gamma}(u) \geqslant K \rho^{2}-C \rho^{2^{*}}
$$

for any $u \in \mathbb{P}_{j}$ with $\|u\|=\rho$. By taking $\rho>0$ small enough, (4.16) gives that

$$
\mathcal{J}_{\gamma}(u) \geqslant \alpha
$$

for a suitable $\alpha>0$, since $2^{*}>2$.

Lemma 4.6. Let $f$ satisfy (1.11).

Then, for any $\gamma>0$ there exists a constant $M>0$, independent of $\gamma$, such that $\max _{u \in \mathbb{H}_{k}} \mathcal{J}_{\gamma}(u)<M$. 
Proof. Fix $\gamma>0$. By (1.11) and (4.9), for any $u \in \mathbb{H}_{k} \backslash\{0\}$ we have

$$
\begin{aligned}
\mathcal{J}_{\gamma}(u) & \leqslant \frac{1}{2}\|u\|^{2}-\frac{\lambda_{k}}{2}\|u\|_{2}^{2}-\frac{\gamma}{2^{*}}\|u\|_{2^{*}}^{2^{*}}+B|\Omega| \\
& \leqslant B|\Omega|-\frac{\gamma}{2^{*}}\|u\|_{2^{*}}^{2^{*}} \\
& <B|\Omega|,
\end{aligned}
$$

concluding the proof of Lemma 4.6,

Proof of Theorem 1.2, By Lemma 3.2, Lemma 4.5 and Lemma 4.6, there is $\gamma^{*}>0$ sufficiently small such that $\mathcal{J}_{\gamma}$ satisfies $\left(I_{2}\right)-\left(I_{4}\right)$ of Theorem 2.1 for any $\gamma \in\left(0, \gamma^{*}\right)$. By recalling that $\mathbb{P}_{j}=H_{j-1}^{\perp}$, we get that codim $\mathbb{P}_{j}=j-1$. Hence, by Theorem 2.1 we conclude that $\mathcal{J}_{\gamma}$ admits $k-j+1$ pairs of non-trivial critical points for any $\gamma \in\left(0, \gamma^{*}\right)$. Then, the proof of Theorem 1.2 is complete.

Remark 4.7. We would like to point out that when $j=1$ we can also replace (1.11) with (1.9) and Theorem 1.2 still holds true. Indeed, we can argue exactly as in the proof of Theorem 1.1, by using Lemma 4.5 (with $\mathbb{P}_{1}=X_{0}^{s}(\Omega)$ ) instead of Lemma 4.2.

4.3. Proof of Theorem 1.3. We first show that problem (1.1) possesses a non-trivial non-negative solution. For this, it is sufficient to study the following problem

$$
\begin{cases}(-\Delta)^{s} u=\gamma u^{2^{*}-1}+\widetilde{f}(x, u) & \text { in } \Omega \\ u \geqslant 0 & \text { in } \Omega \\ u=0 & \text { in } \mathbb{R}^{n} \backslash \Omega,\end{cases}
$$

where

$$
\widetilde{f}(x, t)= \begin{cases}f(x, t) & \text { if } t>0 \\ 0 & \text { if } t \leqslant 0 .\end{cases}
$$

Indeed, a non-trivial solution of (4.17) is a non-trivial non-negative solution of (1.1).

The energy functional associated with (4.17) is given by

$$
\widetilde{\mathcal{J}}_{\gamma}(u)=\frac{1}{2}\|u\|^{2}-\frac{\gamma}{2^{*}} \int_{\Omega}(u(x))^{2^{*}} d x-\int_{\Omega} \widetilde{F}(x, u(x)) d x,
$$

where

$$
\widetilde{F}(x, t)=\int_{0}^{t} \widetilde{f}(x, \tau) d \tau .
$$

We would observe that the truncated function $\tilde{f}$ still verifies (1.3), (11.6), (1.7) and (1.10), while (1.11) holds true for $\tilde{f}$ for any $t \geqslant 0$ but not for any $t<0$. This point must be considered for our proof.

Indeed, in order to apply Theorem 2.2 , we immediately note that $\widetilde{\mathcal{J}}_{\gamma}$ still verifies $\left(I_{2}\right)$ by Lemma 4.5 with $\mathbb{P}_{1}=X_{0}^{s}(\Omega)$. In order to prove $\widehat{\left(I_{3}\right)}$ of Theorem 2.2 we have to proceed as follows.

Let $e_{1}$ be the eigenfunction of $(-\Delta)^{s}$ associated to $\lambda_{1}$. Since $e_{1}$ is positive by [21, Corollary 8], by (4.18) it follows that $\widetilde{F}\left(x, t e_{1}(x)\right)=F\left(x, t e_{1}(x)\right)$ for any $t>0$ and for a.e. $x \in \Omega$. Thus, we can use (1.11) and get for any $t>0$

$$
\begin{aligned}
\widetilde{\mathcal{J}}_{\gamma}\left(t e_{1}\right) & =\frac{1}{2}\left\|t e_{1}\right\|^{2}-\frac{\gamma}{2^{*}}\left\|t e_{1}\right\|_{2^{*}}^{2^{*}}-\int_{\Omega} \widetilde{F}\left(x, t e_{1}(x)\right) d x \\
& \leqslant \frac{t^{2}}{2}\left\|e_{1}\right\|^{2}-\frac{t^{2}}{2} \lambda_{1}\left\|e_{1}\right\|_{2}^{2}+B|\Omega| \\
& =B|\Omega|,
\end{aligned}
$$

thanks to the characterization of $e_{1}$ given in [19, Proposition 9]. From this, $\tilde{\mathcal{J}}_{\gamma}$ satisfies $\widehat{\left(I_{3}\right)}$ for any $\gamma>0$. 
Now it remains to verify $\left(I_{4}\right)$ of Theorem 2.2. for this it is enough to argue as in the proof of Lemma 3.1 and Lemma 3.2 (note that for these lemmas we just need assumptions (1.3), (1.6) and (1.7) ).

Finally, all the assumptions of Theorem 2.2 are satisfied by $\widetilde{\mathcal{J}}_{\gamma}$ and so we can conclude

that for any $\gamma \in\left(0, \gamma^{*}\right), \widetilde{\mathcal{J}}_{\gamma}$ has a non-trivial critical point which is a non-trivial nonnegative solution for (1.1). In a similar way, with small modifications, it is possible to prove the existence of a non-trivial non-positive solution for (1.1). This ends the proof of Theorem 1.3.

\section{REFERENCES}

[1] R.A. Adams, Sobolev Spaces, Academic Press, New York, 1975.

[2] A. Ambrosetti And P. Rabinowitz, Dual variational methods in critical point theory and applications, J. Funct. Anal., 14, 349-381 (1973).

[3] B. Barrios, E. Colorado, R. Servadei And F. Soria, A critical fractional equation with concaveconvex power nonlinearities, Ann. Inst. H. Poincaré Anal. Non Linéaire, 32, 875-900, (2015).

[4] P. Bartolo, V. Benci And D. Fortunato, Abstract critical point theorems and applications to some nonlinear problems with strong resonance at infinity, Nonlinear Anal., 7, 981-1012 (1983).

[5] R. Bartolo And G. Molica Bisci, Asymptotically linear fractional p-Laplacian equations, to appear in Annali Mat. Pura Appl.

[6] H. BrÉzis, Analyse fonctionelle. Théorie et applications, Masson, Paris (1983).

[7] E. Di Nezza, G. Palatucci And E. Valdinoci, Hitchhiker's guide to the fractional Sobolev spaces, Bull. Sci. Math., 7, 981-1012 (1983).

[8] A. Fiscella, G. Molica Bisci And R. Servadei, Bifurcation and multiplicity results for critical nonlocal fractional Laplacian problems, Bull. Sci. Math., 140, 14-35 (2016).

[9] A. Fiscella, R. Servadei And E. Valdinoci, Density properties for fractional Sobolev spaces, Ann. Acad. Sci. Fenn. Math., 40, 235-253 (2015).

[10] A. Fiscella and E. Valdinoci, A critical Kirchhoff type problem involving a nonlocal operator, Nonlinear Anal., 94, 156-170 (2014).

[11] G. Franzina and G. Palatucci, Fractional p-eigenvalues, Riv. Mat. Univ. Parma, 5, $373-386$ (2014).

[12] E. Lindgren And P. Lindqvist, Fractional eigenvalues, Calc. Var., 49, 795-826 (2014).

[13] S. Mosconi And M. Squassina, Nonlocal problems at nearly critical growth, preprint available online at http://arxiv.org/abs/1512.01956.

[14] G. PAlatucci AND A. PISANTE, Improved Sobolev embeddings, profile decomposition, and concentration-compactness for fractional Sobolev spaces, Calc. Var. Partial Differential Equations, 50, no. 3-4, 799-829 (2014).

[15] K. Pereira, M. Squassina And Y. Yang, Bifurcation results for critical growth fractional p-Laplacian problems, Math. Nachr., 289, 332-342 (2015).

[16] R. Servadei, The Yamabe equation in a non-local setting, Adv. Nonlinear Anal., 2, 235-270 (2013).

[17] R. SeRvadei And E. VAldinOci, Lewy-Stampacchia type estimates for variational inequalities driven by (non)local operators, Rev. Mat. Iberoam., 29, no. 3, 1091-1126 (2013).

[18] R. Servadei And E. Valdinoci, Mountain Pass solutions for non-local elliptic operators, J. Math. Anal. Appl., 389, 887-898 (2012).

[19] R. SERVAdei And E. VAldinoci, Variational methods for non-local operators of elliptic type, Discrete Contin. Dyn. Syst., 33, no. 5, 2105-2137 (2013).

[20] R. Servadei and E. Valdinoci, A Brezis-Nirenberg result for non-local critical equations in low dimension, Commun. Pure Appl. Anal., 12, no. 6, 2445-2464 (2013).

[21] R. Servadei And E. VAldinoci, Weak and viscosity solutions of the fractional Laplace equation, Publ. Mat., 58, no. 1, 133-154 (2014).

[22] R. Servadei And E. Valdinoci, The Brezis-Nirenberg result for the fractional Laplacian, Trans. Amer. Math. Soc., 367, no. 1, 67-102 (2015).

[23] E.A.B. Silva AND M.S. XAVIER, Multiplicity of solutions for quasilinear elliptic problems involving critical Sobolev exponents, Ann. Inst. H. Poincaré Anal. Non Linéaire, 20, no. 2, 341-358 (2003). 
A. FISCELLA, G. MOLICA BISCI, AND R. SERVADEI

Departamento de Matemática, Universidade Estadual de Campinas, imeCC, Rua Sérgio Buarque de Holanda 651, SP CEP 13083-859 CAmpinas, BRAZIL

E-mail address: fiscella@ime.unicamp.br

Dipartimento PAU, Università 'Mediterranea' di Reggio Calabria, Via Melissari 24, 89124 Reggio Calabria, Italy

E-mail address: gmolica@unirc.it

Dipartimento di Scienze Pure e Applicate (DiSPeA), Universita degli Studi di Urbino 'Carlo Bo', Piazza della Repubblica 13, 61029 Urbino (Pesaro e Urbino), Italy

E-mail address: raffaella.servadei@uniurb.it 